\title{
Unique Challenges, Solutions and Methodologies for Planetary Sample Analyses
}

\author{
Zia Rahman $^{1 *}$, L. P. Keller ${ }^{2}$, A. N. Nguyen ${ }^{1}$, C. J. Snead ${ }^{1}$ and Scott A. Messanger ${ }^{2}$ \\ 1. Science Department, Jacobs, NASA-JSC, TX, USA. \\ 2. ARES, NASA-JSC, Houston, TX, USA. \\ *Corresponding Author: zia.rahman-1@nasa.gov
}

We have developed innovative focused ion beam (FIB) sample preparation methods that allow us to perform coordinated analyses of planetary materials using combinations of ion microprobe, synchrotron-based instruments, and transmission electron microscopy (TEM) measurements on the same sample. We discuss several examples below utilizing the focused ion beam (FIB), TEM and other instruments at the NASA Johnson Space Center.

The trace element content of mineral grains in comet dust provides important clues on their formation and processing in the early solar system. We preformed coordinated analyses of a comet dust particle that had been prepared using ultramicrotomy for TEM analysis. Problem; extraction of a ultramicrotome thin section from a TEM grid in order to enable other analyses. A carbon ring $\sim 2-3$ um thick was FIB deposited on top of the carbon film, the film near the outer rim of the deposited ring was milled using various patterns to uniformly release the stresses on the film, preventing rupture and collapse, and was attached to the micromanipulator needle (Fig. 1a). The ring was isolated from the film and transferred to a silicon sample holder (Fig. 1b, c) for analysis using the HXN (hard X-ray nanoprobe) beamline at NSLSII Brookhaven National Lab [1].

Rare sub- $\mu \mathrm{m}$ presolar grains that originate in evolved stars and supernovae, occur in primitive astromaterials and are identified by their exotic isotopic compositions [2]. Problem; need to reduce background signals from neighboring grains to obtain accurate isotopic analyses of $\mathrm{Mg}$ and $\mathrm{Fe}$, using the NanoSIMS. We utilized FIB milling to remove surrounding contaminant grains by precisely depositing a protective cap of Pt on top of the $\sim 200 \mathrm{~nm}$ grain and then milled $\sim 5 \mu \mathrm{m}$ diameter of the surrounding material (Fig. 2a). Following the isotopic analyses, the spindle was extracted and thinned to electron transparency for TEM analyses (Fig. 2b, c).

Microparticles removed from cosmic dust collectors have traditionally been stored and distributed to investigators in glass concavity slides [3]. Problem; sample electrostatic charging due to friction between the slides and particles. We developed a solution to replace the glass slides with a chargedissipative substrate ( $\mathrm{Si}$ or $\mathrm{Ge}$ ). Particles retain a high level of visibility, triboelectric charging is significantly reduced enabling reliable manipulation in ambient atmospheric conditions. We utilized FIB to mill several shallow $(<20 \mu \mathrm{m})$ depressions $\sim 30 \mu \mathrm{m}^{2}$ on Si and Ge chips (Fig. 3a, b). A $10 \mu \mathrm{m}$ particle of a CM2 meteorite was placed into one of the wells using a micromanipulator (Fig. $3 \mathrm{c}$ ). The charge-dissipative nature of Si chip enabled successful acquisition of secondary electron image of the stored particle; highly desirable as these storage substrates minimize the need for high-risk microparticle transfers between storage and analysis substrates.

\section{References:}

[1] GJ Flynn et al., Meteoritics Planet. Sci. (2016) \#6205. 
[2] AN Nguyen et al., Astrophysical J. 818 (2016), p. 1.

[3] CJ Snead et al., Hayabusa Symposium, Tokyo (2018).
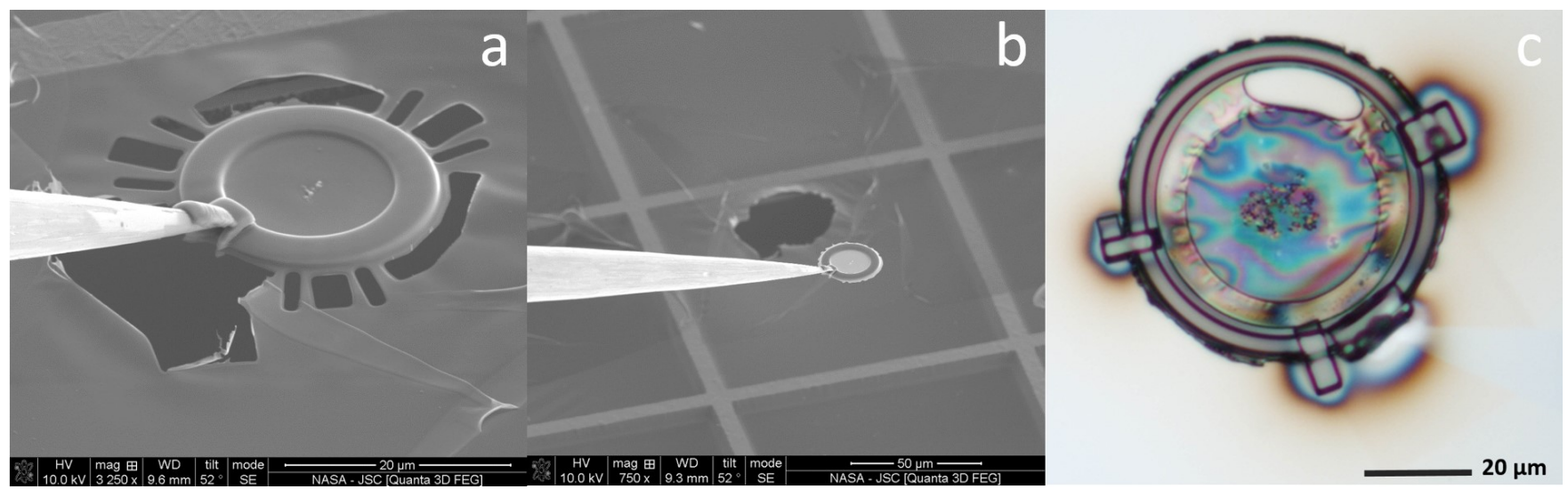

Figure 1. a) thin section with deposited C-ring attached to the needle. b) assembly lifted from the C film/TEM grid. c) reflected light image of the extracted section attached to a Si substrate for analysis.

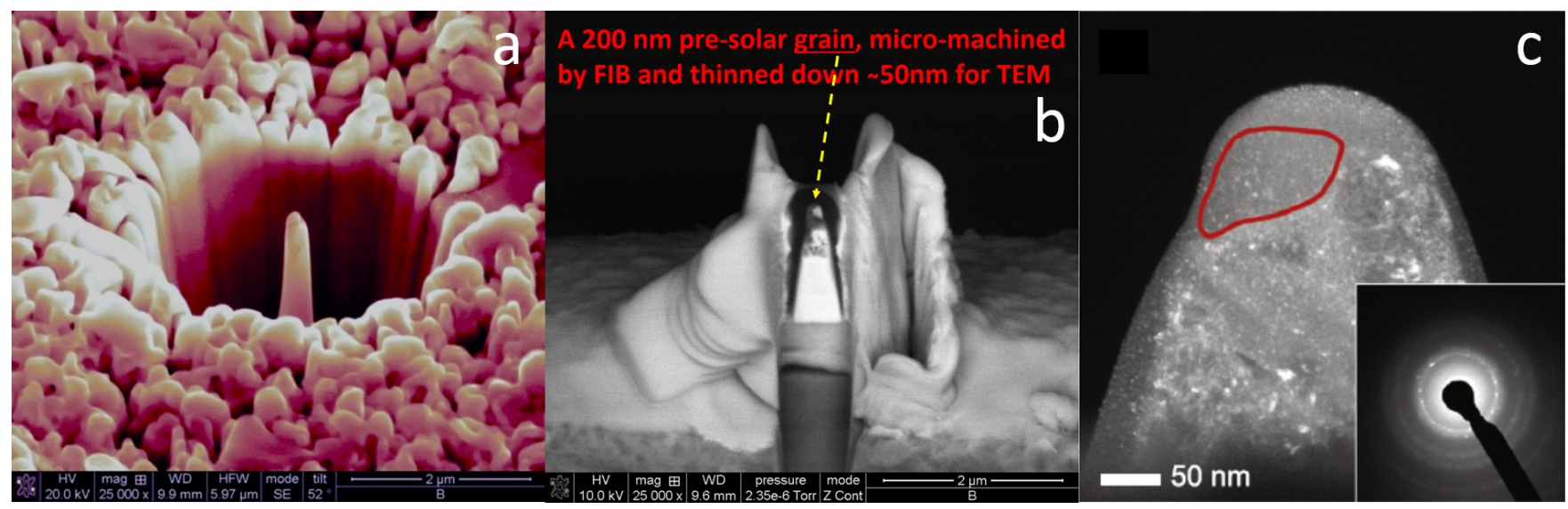

Figure 2. a) a sub- $\mu \mathrm{m}$ presolar grain is isolated from neighboring meteorite matrix grains by FIB milling for subsequent NanoSIMS isotopic analysis. b) an electron transparent section of the grain was then produced by FIB for mineralogical analysis by TEM. c) darkfield TEM image and diffraction pattern from the presolar grain [3].

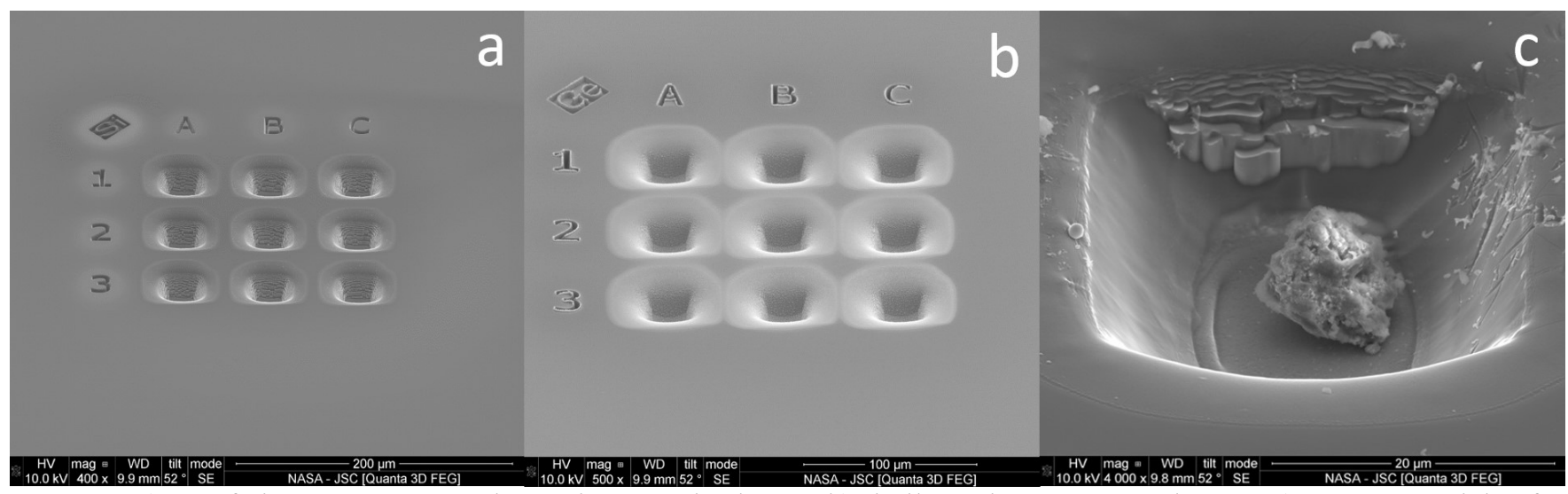

Figure 3. a) set of nine 30×30x10 um depressions on Si substrate b) similar as in 'a' on Ge substrate. c) a 10 um particle of a CM2 meteorite in one of the cavities. 